\title{
WEAK HYPERBOLICITY ON PERIODIC ORBITS FOR POLYNOMIALS.
}

\author{
JUAN RIVERA-LETELIER
}

\begin{abstract}
We prove that if the multipliers of the repelling periodic orbits of a complex polynomial grow at least like $n^{5+\varepsilon}$, for some $\varepsilon>0$, then the Julia set of the polynomial is locally connected when it is connected. As a consequence for a polynomial the presence of a Cremer cycle implies the presence of a sequence of repelling periodic orbits with "small" multipliers. Somehow surprisingly the proof is based in measure theorical considerations.
\end{abstract}

Consider a polynomial $P$ with complex coefficients. Given a periodic point $p \in \mathbb{C}$ of $P$ of minimal period $n \geq 1$, we call $\lambda=\left(P^{n}\right)^{\prime}(p)$ the multiplier of $p$. We say that $p$ is repelling, indifferent or attracting if $|\lambda|>1,|\lambda|=1$ or $|\lambda|<1$, respectively.

Recall that the set,

$$
K(P)=\left\{z \in \mathbb{C} \mid\left\{P^{n}(z)\right\}_{n \geq 1} \text { is bounded }\right\},
$$

is called the filled-in Julia set of $P$ and its boundary is called the Julia set of $P$, which is denoted by $J(P)$.

Our main result is the following.

Theorem 1. Let $P \in \mathbb{C}[z]$ be a polynomial with connected Julia set. Suppose that there are constants $C>0$ and $\varepsilon>0$ such that for every repelling periodic point $p \in \mathbb{C}$ of period $n$,

$$
\left|\left(P^{n}\right)^{\prime}(p)\right| \geq C n^{5+\varepsilon} .
$$

Then the Julia set of $P$ is locally connected.

In fact we prove the stronger statement that $\overline{\mathbb{C}}-K(P)$ is an integrable domain in the sense of $[5]$.

The class of polynomial (and rational) maps for which the multipliers grow exponentially in the period is studied in [14] and the proof of Theorem 11 is based on a variant of Lemma 2.1 of that paper.

Recall that a Cremer cycle is a cycle for which the polynomial is not locally linearizable and whose multiplier is not a root of unity. By a theorem of A. Douady and D. Sullivan the Julia set of polynomial with a Cremer cycle is not locally connected, see [18]. So the following corollary follows directly from Theorem 1 .

Corollary 1. Let $P \in \mathbb{C}[z]$ be a polynomial with connected Julia set and with a Cremer cycle. Then for every $\varepsilon>0$ there is a sequence $\left\{p_{k}\right\}$ of repelling periodic

Date: October 8, 2001. 
points of $P$ of period $n_{k}$ satisfying,

$$
\left|\left(P^{n_{k}}\right)^{\prime}\left(p_{k}\right)\right| \leq n_{k}^{5+\varepsilon} .
$$

The corollary remains true if the Julia set is disconnected (see Remark 2) and there is an analogous statement for general rational maps, see Remark 3. Unfortunately this result does not give information about the location of these periodic points. Under certain conditions Cremer cycles imply the existence of the so called "small" cycles; see [19] and [11].

Remark 1. It follows by 22] (Corollary 1.1) that the hypothesis of Theorem I are satisfied for polynomials such that for every critical value $v \in J(P)$ the derivatives $\left|\left(P^{n}\right)^{\prime}(v)\right|$ grow at least as $n^{\alpha}$, for some $\alpha>1$ only depending on the degree of $P$. Such polynomials also satisfy the so called summability condition, see also [10], 13, [1], 16] and 17].

Remark 2. To see that Corollary 1 applies for polynomials with disconnected Julia set we first observe that the proof of the theorem applies for polynomial like maps in the sense of [3]. Then if $P \in \mathbb{C}[z]$ is a polynomial with a Cremer periodic point $p \in \mathbb{C}$ of period $q \geq 1$, then the restriction of $P^{q}$ to a suitable neighborhood of $p$ is a polynomial like map with connected Julia set.

Remark 3. A similar method allows to prove that if a rational map $R \in \mathbb{C}(z)$ has a Cremer cycle, then there is a sequence of periodic points $\left\{p_{k}\right\}$ of period $n_{k}$, whose multiplier is bounded by $\exp \left(C \sqrt{n_{k}}\left(\ln n_{k}\right)^{\frac{3}{2}+\frac{2}{h}}\right)$, where $C>0$ is a universal constant and $h>0$ is any number satisfying $h<H D_{\text {hyp }}(R)$. The latter is the supremum over the Hausdorff dimensions of hyperbolic sets of $R$.

\section{Proof of the theorem.}

Fix a polynomial $P \in \mathbb{C}[z]$ of degree $d>1$ and with connected Julia set. Consider a base point $w_{0} \in \mathbb{C}-K(P)$ to be chosen in Lemma 2 below.

Theorem 1 will be reduced to the following lemma, see [5] for its proof.

Lemma 1. Let $\left\{\omega_{n}\right\}_{n \geq 1}$ be an increasing sequence such that $\sum_{n \geq 1} \omega_{n}^{-1}<\infty$. If for every $n \geq 1$ and every $z \in P^{-n}\left(w_{0}\right)$ we have $\left|\left(P^{n}\right)^{\prime}(z)\right| \geq \omega_{n}$, then $J(P)$ is locally connected.

Let $\left\{\lambda_{n}\right\}_{n \geq 1}$ be an increasing sequence and suppose that for every $n \geq 1$ the repelling periodic points of period $n$ have multipliers of norm at least $\lambda_{n}>0$.

The following lemma estimates the derivative at a good portion of preimages of a base point $w_{0} \in \mathbb{C}-K(P)$. Lemmas 3 and 4 are distortion lemmas that will allow us to estimate derivatives at all preimages of $w_{0}$, as required by Lemma if.

Lemma 2. Let $\mu$ be an invariant probability measure with positive entropy $h_{\mu}$ supported on a hyperbolic set $K \subset J(P)$. Then there is a base point $w_{0} \in \mathbb{C}-K(P)$, a constant $C_{0}=C_{0}\left(w_{0}\right)>0$ and a non-decreasing sequence of integers $\{\ell(k)\}_{k \geq 1}$ such that the following properties hold. 
1. There is $L=L(K) \geq 1$ such that $\ell(k+1)-\ell(k) \leq L$, for $k \geq 1$. Moreover for every $\varepsilon_{0}>0$ we have $\ell(k) \leq\left(h_{\mu}^{-1}+\varepsilon_{0}\right) \ln k$, for $k$ big enough.

2. For every $k \geq 1$ there is a point $x \in P^{-\ell(k)}\left(w_{0}\right)$ such that for every $w^{\prime} \in$ $P^{-k}(x)$,

$$
\left|\left(P^{k+\ell(k)}\right)^{\prime}\left(w^{\prime}\right)\right| \geq C_{0} \lambda_{k+\ell(k)} .
$$

Proof. Since $K$ is a hyperbolic set the following well-known univalent pull-back property holds. There is $\delta>0$ such that for every $z_{0} \in K$ there is $\zeta_{0} \in K$ such that for every $r>0$ small there is a univalent pull-back $V \subset B\left(z_{0}, r\right)$ of $B\left(\zeta_{0}, 3 \delta\right)$ whose diameter is comparable to $r$. Let $R>1$ such that for any $r>0$ small and any univalent pull-back of $B\left(z_{0}, R r\right)$, the corresponding pull-back of $B\left(z_{0}, r\right)$ has diameter at most $\delta$.

Note that for small $r>0$ any pull-back of $B\left(z_{0}, r\right)$ will be contained in a definite neighborhood $U$ of $J(P)$. Let $m_{0} \geq 1$ be such that for every $\zeta_{0} \in J(P)$ the set $\cup_{0 \leq m \leq m_{0}} P^{m}\left(B\left(\zeta_{0}, \delta\right)\right)$ contains $U$.

1.- By Ruelle's inequality $\chi_{\mu}=\int\left|P^{\prime}\right| d \mu \geq \frac{1}{2} h_{\mu}>0$ is positive and then $H D(\mu)=\frac{h_{\mu}}{\chi_{\mu}}$ is also positive, where $H D(\mu)$ denotes the infimum of the Hausdorff dimensions of sets $X \subset K$ such that $\mu(X)=1$; see [9] or [15.

2.- Choose $\alpha>H D(\mu)^{-1}$. By a Borel-Cantelli argument for every $z_{0} \in K$, outside an exceptional set of Hausdorff dimension at most $\alpha^{-1}<H D(\mu)$, there is $k_{0}=k_{0}\left(z_{0}\right)$ such that for all $k \geq k_{0}$ the ball $B\left(z_{0}, R k^{-\alpha}\right)$ is disjoint form $\cup_{0 \leq i \leq k+m_{0}} P^{i}$ (Crit); here Crit $\subset \mathbb{C}$ denotes the set of critical points of $P$.

So for big values of $k$ all pull-backs of $B\left(z_{0}, R k^{-\alpha}\right)$ by $f^{k+m_{0}}$ are univalent.

3.- Since $\alpha^{-1}<H D(\mu)$ it follows that the set of such points $z_{0} \in K$ has full $\mu$ measure. On the other hand it follows by Birkhoff Ergodic Theorem there is a set of $\mu$ positive measure of points in $K$ whose Lyapunov exponent is at least $\chi_{\mu}=\int\left|P^{\prime}\right| d \mu$, cf. [15].

Thus there is such a point $z_{0} \in K$ having Lyapunov exponent at least $\chi_{\mu}$.

4.- Let $\zeta_{0} \in K$ be the corresponding point as explained above and choose any point $w_{0} \in B\left(\zeta_{0}, \delta\right)-K(P)$ as a base point.

For $k \geq 1$ let $\ell(k)$ be an integer such that there is a univalent pull-back $V \subset$ $B\left(z_{0}, k^{-\alpha}\right)$ of $B\left(\zeta_{0}, 3 \delta\right)$ by $P^{\ell(k)}$ with $\operatorname{diam}(V)$ comparable to $k^{-\alpha}$. We assume that $\{\ell(k)\}_{k \geq 1}$ is a non-decreasing sequence. Note that $\ell(k+1)-\ell(k) \leq L$, for $L \geq 1$ only depending on $K$.

5.- Choose $\lambda \in\left(1, \exp \left(\chi_{\mu}\right)\right)$. Since the Lyapunov exponent of $z_{0}$ is at least $\chi_{\mu}$ it follows that for $k$ big, $\ell(k) \leq \frac{\alpha}{\ln \lambda} \ln k$. Moreover for every $\varepsilon_{0}>0$ we can choose $\alpha$ and $\lambda$ close enough to $H D(\mu)^{-1}$ and $\exp \left(\chi_{\mu}\right)$ respectively, so that

$$
\frac{\alpha}{\ln \lambda} \leq \frac{H D(\mu)^{-1}}{\chi_{\mu}}+\varepsilon_{0}=h_{\mu}^{-1}+\varepsilon_{0} .
$$

6.- Fix a big integer $k \geq 1$ and let $V \subset B\left(z_{0}, k^{-\alpha}\right)$ be a univalent pull-back of $B\left(\zeta_{0}, 3 \delta\right)$ by $f^{\ell(k)}$ and let $x \in V$ be the corresponding preimage of $w_{0} \in B\left(\zeta_{0}, \delta\right)$. Let $w^{\prime} \in P^{-k}(x)$ and let $w^{\prime \prime} \in B\left(\zeta_{0}, \delta\right)$ a preimage of $w^{\prime}$ by $P^{m}$, for some $0 \leq m \leq m_{0}$. Let $V^{\prime}$ and $V^{\prime \prime}$ be the pull-backs of $V$ by $f^{k}$ and $f^{k+m}$ containing $w^{\prime}$ and $w^{\prime \prime}$ respectively. Since the corresponding pull-backs of $B\left(z_{0}, R k^{-\alpha}\right)$ are univalent, it follows that $\operatorname{diam}\left(V^{\prime \prime}\right) \leq \delta$ and therefore $V^{\prime \prime} \subset B\left(\zeta_{0}, 2 \delta\right)$. Thus $V^{\prime \prime}$ contains a repelling periodic point of period $k+\ell(k)+m$. By Koebe Distortion Theorem there is a universal constant $K_{1}>0$ such that $\left|\left(P^{k+\ell(k)+m}\right)^{\prime}\left(w^{\prime \prime}\right)\right| \geq K_{1} \lambda_{k+\ell(k)+m}$. 
Thus letting $M=\sup _{U}\left|P^{\prime}\right|$ we have,

$$
\begin{aligned}
\left|\left(P^{k+\ell(k)}\right)^{\prime}\left(w^{\prime}\right)\right| & \geq M^{-m_{0}}\left|\left(P^{k+\ell(k)+m}\right)^{\prime}\left(w^{\prime \prime}\right)\right| \\
& \geq K_{1} M^{-m_{0}} \lambda_{k+\ell(k)+m} \geq K_{1} M^{-m_{0}} \lambda_{k+\ell(k)} .
\end{aligned}
$$

Let $\mathbb{H} \subset \mathbb{C}$ be the upper half plane and consider a covering map $\psi: \mathbb{H} \longrightarrow$ $\mathbb{C}-K(P)$ with deck transformation $z \longrightarrow z+1$ and such that $P(\psi(z))=\psi(d z)$. In particular $\psi(z+1)=\psi(z)$, for $z \in \mathbb{H}$, and for any $r \in \mathbb{R}, \psi$ is injective in $\{z \in \mathbb{H} \mid r<\operatorname{Re}(z)<r+1\}$. The following are distortion lemmas.

Lemma 3. There is a constant $D>1$ such that for all $\tilde{w}$ and $\tilde{w}^{\prime} \in \mathbb{H}$ satisfying $|\operatorname{Im}(\tilde{w})|=\left|\operatorname{Im}\left(\tilde{w}^{\prime}\right)\right| \leq\left|\tilde{w}-\tilde{w}^{\prime}\right| \leq 1 / 2$,

$$
\left|\psi^{\prime}(\tilde{w})\right| \leq D\left(\left|\tilde{w}-\tilde{w}^{\prime}\right| /|\operatorname{Im}(\tilde{w})|\right)^{4}\left|\psi^{\prime}\left(\tilde{w}^{\prime}\right)\right| .
$$

Proof. Put $\rho=\operatorname{Re}\left(\frac{\tilde{w}+\tilde{w}^{\prime}}{2}\right), s=\left|\tilde{w}-\tilde{w}^{\prime}\right| \leq 1 / 2$ and $h=\operatorname{Im}(\tilde{w})=\operatorname{Im}\left(\tilde{w}^{\prime}\right) \leq s$. Note that $\psi$ is univalent in the square

$$
S=\{z \in \mathbb{H}|| \operatorname{Re}(z)-\rho \mid<s \text { and } \operatorname{Im}(z)<2 s\} \subset\{z \in \mathbb{H}|| \operatorname{Re}(z)-\rho \mid<1 / 2\}
$$

Moreover by hypothesis $\tilde{w}, \tilde{w}^{\prime} \in S_{0}=\{z \in \mathbb{H}|| \operatorname{Re}(z)-\rho \mid \leq s / 2$ and $\operatorname{Im}(z) \leq s\}$.

Let $\varphi: S \longrightarrow \mathbb{D}$ be a conformal representation. By Schwarz' reflection principle $\varphi$ extends unvalently to $\{z \in \mathbb{C}|| \operatorname{Re}(z)-\rho \mid<s$ and $|\operatorname{Im}(z)|<2 s\}$. We normalize $\varphi$ in such a way that $\varphi(\rho)=-i$ and such that the interval $I=(\rho-s, \rho+s) \subset \mathbb{R} \subset \mathbb{C}$ is mapped to the semi circle $\{\exp (i \theta) \mid \pi<\theta<2 \pi\}$.

Since $\tilde{w}, \tilde{w}^{\prime} \in S_{0}$ and $I \subset \bar{S}_{0}$ it follows by Koebe distortion theorem that there is a constant $K>1$ such that $K^{-1}\left|\varphi^{\prime}(\tilde{w})\right| \leq\left|\varphi^{\prime}\left(\tilde{w}^{\prime}\right)\right| \leq K\left|\varphi^{\prime}(\tilde{w})\right|$ and such that $\widehat{w}=\varphi(\tilde{w}), \widehat{w}^{\prime}=\varphi\left(\tilde{w}^{\prime}\right) \in\left\{|z|<1-K^{-1} s^{-1} h\right\}$.

By Theorem 1.3 of $\left[12\right.$ applied to $\psi \circ \varphi^{-1}: \mathbb{D} \longrightarrow \mathbb{C}$ there is a universal constant $K_{1}>1$ such that,

$$
K^{-2}\left|\psi^{\prime}(\tilde{w})\right| /\left|\psi^{\prime}\left(\tilde{w}^{\prime}\right)\right| \leq\left|\left(\psi \circ \varphi^{-1}\right)^{\prime}(\widehat{w})\right| /\left|\left(\psi \circ \varphi^{-1}\right)^{\prime}\left(\widehat{w}^{\prime}\right)\right| \leq K_{1}\left(K^{-1} s^{-1} h\right)^{-4},
$$

so the lemma follows with $D=K_{1} K^{6}$.

Lemma 4. There is a constant $C_{1}=C_{1}\left(w_{0}\right)>1$ such that the following property holds. Consider an integer $\ell \geq 1$ and $x \in P^{-\ell}\left(w_{0}\right)$. Then for every integer $k \geq 1$ and every $w \in P^{-(k+\ell)}\left(w_{0}\right)$ there is $w^{\prime} \in P^{-k}(x)$ such that,

$$
\left|\left(P^{k+\ell}\right)^{\prime}(w)\right| \geq C_{1} d^{-4 \ell}\left|\left(P^{k+\ell}\right)^{\prime}\left(w^{\prime}\right)\right| .
$$

Proof. Let $\tilde{w}_{0}$ and $\tilde{w} \in \mathbb{H}$ be such that $\psi\left(\tilde{w}_{0}\right)=w_{0}$ and $\psi(\tilde{w})=w$. We may choose $\tilde{x} \in \mathbb{H}$ such that $\psi(\tilde{x})=x$ and $\left|\tilde{x}-d^{k} \tilde{w}\right| \leq 1$. Put $\tilde{w}^{\prime}=d^{-k} \tilde{x}$ and $w^{\prime}=\psi\left(\tilde{w}^{\prime}\right) \in P^{-k}(x)$. Since $\operatorname{Im}\left(\tilde{w}^{\prime}\right)=d^{-(k+\ell)} \operatorname{Im}\left(\tilde{w}_{0}\right)=\overline{\operatorname{Im}}(\tilde{w})$ the previous lemma implies that $\left|\psi^{\prime}(\tilde{w})\right| \leq D \operatorname{Im}\left(\tilde{w}_{0}\right)^{-4} d^{4 \ell}\left|\psi^{\prime}\left(\tilde{w}^{\prime}\right)\right|$.

On the other hand form the equation $P(\psi(z))=\psi(d z)$ it follows that for every $\widehat{w} \in \mathbb{H}$ such that $P^{k+\ell}(\psi(\widehat{w}))=w_{0}$,

$$
\left(P^{k+\ell}\right)^{\prime}(\psi(\widehat{w})) \psi^{\prime}(\widehat{w})=d^{k+\ell} \psi^{\prime}\left(d^{k+\ell} \widehat{w}\right)=d^{k+\ell} \psi^{\prime}\left(w_{0}\right),
$$

which only depends on $w_{0}$. So the lemma follows with $C_{1}=D^{-1} \operatorname{Im}\left(\tilde{w}_{0}\right)^{4}$.

Note that as the invariant measure $\mu$ is chosen with bigger entropy $h_{\mu}$, the (asymptotic) estimate of Lemma 2 is better. The topological entropy of $P$ is equal 
to $\ln d$ (see [6] and [8]), so by the variational principle $h_{\mu} \leq \ln d$; cf. [15]. On the other hand the harmonic measure of $P$ is invariant under $P$ and it has metric entropy equal to $\ln d$, see [1] and also [1] and [7. It follows by Pesin theory that we can choose an invariant measure $\mu$ supported on a hyperbolic set and such that $h_{\mu}$ is as close to $\ln d$ as wanted, see [15].

Proof of the Theorem. Put $\lambda_{n}=C n^{5+\varepsilon}$ and let $\varepsilon_{0}>0$ be such that $(4 \ln d) \varepsilon_{0}<$ $\varepsilon / 3$. Let $\mu$ be an invariant probability measure supported on a hyperbolic set and whose metric entropy $h_{\mu}$ is close enough to $\ln d$ so that $(4 \ln d) h_{\mu}^{-1}<4+\varepsilon / 3$. Let $w_{0} \in \mathbb{C}-K(P), C_{0}=C_{0}\left(w_{0}\right)>0$ and $\{\ell(k)\}_{k \geq 1}$ be given by Lemma 2. Moreover let $n \geq 1$ big and $z \in P^{-n}\left(w_{0}\right)$.

Then there is $k$ such that $n \leq k+\ell(k) \leq n+L+1$. Let $w \in P^{-(k+\ell(k)-n)}(z) \subset$ $P^{-(k+\ell(k))}\left(w_{0}\right)$ and note that $\left|\left(P^{n}\right)^{\prime}(z)\right| \geq M^{-(L+1)}\left|\left(P^{k+\ell(k)}\right)^{\prime}(w)\right|$, where $M=$ $\sup \left|P^{\prime}\right|$ taken over all preimages of $w_{0}$.

Let $x \in P^{-\ell(k)}\left(w_{0}\right)$ be given by Lemma 2 and let $w^{\prime} \in P^{-k}(x)$ be given by Lemma 4 so that,

$$
\left|\left(P^{k+\ell(k)}\right)^{\prime}(w)\right| \geq C_{1} d^{-4 \ell(k)}\left|\left(P^{k+\ell(k)}\right)^{\prime}\left(w^{\prime}\right)\right| \geq C_{0} C_{1} d^{-4 \ell(k)} \lambda_{k+\ell(k)} .
$$

By part 1 of Lemma 2, if $k$ is big enough $d^{4 \ell(k)} \leq k^{4 \ln d\left(h_{\mu}^{-1}+\varepsilon_{0}\right)} \leq k^{4+2 \varepsilon / 3}$. Thus

$\left|\left(P^{n}\right)^{\prime}(z)\right| \geq M^{-(L+1)}\left|\left(P^{k+\ell(k)}\right)^{\prime}(w)\right| \geq M^{-(L+1)} C_{0} C_{1} \lambda_{k+\ell(k)} k^{-(4+2 \varepsilon / 3)} \geq C_{2} n^{1+\varepsilon / 3}$,

where $C_{2}=M^{-(L+1)} C C_{0} C_{1}$. Thus the hypothesis of Lemma 11 is satisfied with $\omega_{n}=C_{2} n^{1+\varepsilon / 3}$.

Acknowledgements. I'm grateful to F. Przytycki and S. Smirnov for several remarks and comments.

\section{REFERENCES}

[1] H. Brolin. Invariant sets under iteration of rational functions. Ark. Mat. 6 (1965), 103-144.

[2] H. Bruin, S. van Strein. Expansion of derivatives in one dimensional dynamics. Preprint September 2000.

[3] A. Douady and J. Hubbard. On the dynamics of polynomial-like mappings. Ann. Sci. Ec. Norm. Sup. 18 (1985), 287-344.

[4] A. Freire, A. Lopes, R. Mañé. An invariant measure for rational maps. Bol. Soc. Bras. Mat. 14 (1983), 45-62.

[5] J. Graczyk, S. Smirnov. Weak expansion and geometry of Julia sets. March 1999 version.

[6] M. Gromov. On the entropy of holomorphic maps. Preprint 1978.

[7] M. Ljubich. Entropy properties of rational endomorphisms of the Riemann sphere. Ergodic Theory Dynam. Systems 3 (1983), 351-385.

[8] M. Misiurewicz, F. Przytycki. Topological entropy and degree of smooth mappings. Bull. Acad. Polon. Sci. 25 (1977), 573-574.

[9] R. Mañé. The Hausdorff dimension of invariant probabilities of rational maps. Dynamical systems, Valparaiso 1986, 86-117, LMN 1331, Springer 1988.

[10] T. Nowicki, S. van Strein. Absolutely continuous invariant measures under the summability condition. Invent. Math. 105 (1991), 123-136.

[11] R. Perez-Marco. Sur les dynamiques holomorphes non linéariisables et une conjecture de V.I. Arnol'd. Ann. Sci. École Norm. Sup. (4) 26 (1993), 565-644.

[12] Ch. Pommerenke. Boundary Behavior of conformal maps. Springer-Verlag, Berlin, 1992.

[13] F. Przytycki. Iterations of holomorphic Collet-Eckmann maps: Conformal and Invariant measures. Appendix: On non-renormalizable quadratic polynomials. Trans. A.M.S. 350 (1998), 717-742. 
[14] F. Przytycki, J. Rivera-Letelier, S. Smirnov. Equivalence and topological invariance of conditions for non-uniform hyperbolicity in iteration of rational maps. Preprint 2000.

[15] F. Przytycki, M. Urbański. Fractals in the plane - the ergodic theory methods. Cambridge University Press, to appear.

[16] F. Przytycki, M. Urbański. Porosity of Julia sets of non-recurrent and parabolic Collet Eckmann functions. Ann. Acad. Sci. Fenn. Math. 26 (2001), 125-154.

[17] J. Rivera-Letelier. Rational maps with decay of geometry: Rigidity, Thurston's algorithm and local connectivity. Preprint IMS at Stony Brook \#2000/9.

[18] D. Sullivan. Conformal dynamical systems. Geometric dynamics, Rio de Janeiro 1981, 727752, LMN 1007, Springer 1983.

[19] J.C. Yoccoz. Petits diviseurs en dimension 1. Astérisque No. 231, 1995.

J. Rivera-Letelier, Mathematics Department, SUny at Stony Brook, Stony Brook, NY 11794-3660

E-mail address: rivera@@math.sunysb.edu 